Monatsschrift f. Geburtshülfe u. Gynäkologie 1923;63:59-60

\title{
An die Mitglieder der Deutschen Gesellschaft für Gynäkologie!
}

Wegen der unsicheren politischen Lage an der Westgrenze des Reich es erscbeint dieses Kundschreiben später als in früheren Jahren. Alle für die Vorbereitung der Heidelberger Tagung in Frage kommenden Einzelheiten naüssen jetzt rasch erledigt werden.

Die Teünahme an der Tagung muß bis zum 1. Mai 1923 dem 2. Schriftführer, Professor Eymer, Heidelberg, Universitäts-Frauenklinik, bekanntgegeben werden. Nur auf besonderes Ersuchen wird Quartier besorgt. Da Heidelberg in der Pfingstwoche von Fremden stark besucht wird, ist baldige Quartier-be3tellung unter Angabe der Ankunftszeit $\beta$ rforderlicb. Die bereitgestellte Wohnung ist im Y"erkehrsbüro, gegenüber dem Babnhof, Anlage, zu erfahren. Dort werden auch sonstige Auskünf te jeder Art erteilt. Sind Quartierbesteller am Erscbeinen verhindert, muß die Wohnung bis spätestens 12. Mai abbestellt werden. Bei späte $\Gamma \beta \mathrm{r} A b s a g \theta$ bringen die Gastwirte die bestellten Tage in Anrechnung.

Mitglieäsneuanmeĩãungen sind bis spätestens 14 Tage vor Beginn der Tagung an den 1. Schriftführer, Professor E. Martin, Elberfeld, Hebammen-lehranstalt, zu riohten.

Die neuen Mitglieder haben den Mitglieds- und Kongreßbeitrag von Mk. 2000.- in Heidelberg im Büro der Versammlung (Neues Kollegienhaus) oder durch Banküberweisung oder mittels Postscheckzahlkarte (Postscheck-konto Köln 4155) an das Konto der Gesellschaît bei der Dßutschen Bank, Zweigstelle Bonn, Kaiserplatz 9, zu bezahlen. Die als Mitgliedskart $\beta$ geltende Quittung ist bei Beginn der Tagung im Büro der Versammlung erhältlich.

Gastteünehmern wird die Teilnehmerkarte gegen Zahlung einer Teil-nehmergebühr von Mk. 2000.- im Versammlungsbüro (Neues Kollegienhaus) ausgehändigt.

Der Zutritt zu den Gßsellschaftssitzungen ist nur gegen Yorzeigen der Mitglieds- oder Gastteilnehmerkarte gestattet.

Die Vo'rtrags- unã DemonstrationsordnungTvïrå demnäohst bekanntgegeben. Die Dauer eines Vortrags darf die Zeit von 10, die Dauer einer Demonstration die Zeit von 5 Minuten nicht überschreiten. Die Vortragenden und Demon-strierenden werden dringend ersucht, das für den Druck bestimmte, möglichst kurze Manuskript unmittelbar nach dem Vortrag oder der Demonstration dem 1. Schriftführer zu übergeben. Anmeldungen zu Diskussionsbemerkungen müssen unter Angabe des Namens und des Wohnortes schriftlich dem Vor-sitzenden übergeben werden. Zettel zu Diskussionsanmeldungen liegen auf dem Vorstandstische bereit.

Diskussionsbemerkungen müssen sogleich nieder-geschrieben und vor Schluß der Tagung dem 1. Schrif tf übrer übergeben werden. Liegt bis spätestens 14 Tage nach Schluß des Kongresses kein Manuskript vor, so unterbleibt die Drucklegung in dem Verhandlungsbericht. Von der Mitwirkung eines Stenographen muß wegen der hohen Kosten abgesehen werden.

Die bisherigen Mitglieder werden ersucht, den Mitglieds- und Kongreßbeitrag von Mk. 2000.durch Banküberweisung oder unter Benutzung von Postscheckzahlkarte mit deutlicher und vollständiger Anschrift an das Konto der Gesellschaît bei der Deutschen Bank, Zweigstelle 
Bonn, Kaiserplatz 9, möglichst sofort einzusenden. Die als Mitgliedskarte geltende Quittung wird dann zugesandt. Mitgliedsbeiträge, welche bis zum 1. Juli 1923 nicht $\mathrm{t}>\mathrm{O}$ An die Mitglieder der Deutschen Gesellschaffc für Gynäkologie!

entricbtet sind, werden unter Übersendung der Quittung durcb Poßtnach-nahme erhoben. Nichtannahnie dieser Sendung gilt a]s $\Delta$ ustrittserklärung.

Ausländer haben den Mitgliedsbeitrag oder die Gastteilnehmergebühr in der gleicben Höbe zu entricbten, die sie vor 1914 in ihrer Landes-währung zu entricbten hatten ( 20 Goldniark $=25$ Frank en $\approx 1$ englisches Pfund $=5$ amerikaniscbe Dollar $\approx 12$ bolländisch $\beta$ Gulden $\approx \approx 20$ nordische Kronen = 25 Lire usw.). Angebörige des österreicbischen, ungariscben und polnischen Staates haben wie die E, eicbsdeutschen Mk. 2000.- zu entrichten.

Schriftlicbe Anfragen werden nur gegen Zusendung des vollen B, ück-portos beantwortet. Adressenänderungen von Mitgliedern sind dem 1. Schriítführer umgehend mitzuteilen. Wer für Demonstrationen Instrumente, Scbalen, Mikroskope und dergl. mehr benötigt, wird gebeten, sicb mit seinen Wünschen bis spätestens 10. Mai 1923 an Herrn Dr. med. Uter, Heidelberg, Universitäts-Frauenklinik, zu -weuden. Präparate und Instrumente können mit Angabe ihrer Bestim-mung vorher an Dr. Uter eingesandt werden. Es steht nur ein Epidiaskop zur Verfügung. Die Projektionsbilder sind numeriert unmittelbar vor dem Vortrag resp. der Demonstration bei dem den Apparat bedienenden Techniker abzugeben.

Kranke, welcbe vorgestellt werden sollen, können, wenn sie 14 Tage vor dem Beginn der Tagung angemeldet sind, in der Universitäts-Frauenklinik untergebracbt werden.

Für die $\Lambda$ usstellung von nicht zu umfangreichen Apparaten und In-strumenten, welche keine besondere Montierung erfordern, stehen neben dem Sitzungssaal ßäume zur Verfügung.

Aussteller wollen sich mit Herrn Hof-mann, Hausmeister des Neuen Kollegienhauses, vorher in Verbindung setzen.

Die Eröffnung der Tagung findet Mittwoch, den 23. Mai 1923, um 9 Uhr vormittags im großen Saal des Neuen Kollegienhauses der Universität statt. Die endgültige Tagesordnung ist im Versammlungsbüro erhältlich.

Am Dienstag, den 22. Mai 1923, begrüßen sich die Teilnehmer um 9 Uhr abends, bei günstiger Witterung im Stadtgarten (Anlage), bei ítegenwetter im Restaurant der Stadthalle

(Neckarstaden). Näheres bierüber ist im Ver-kehrsbüro, gegenûber dem Bahnhof, oder im Versammlungsbüro, Neues Kollegienhaus, oder in der Frauenklinik zu erfahren.

Wegen der politischen Verbältnisse ist es z. Z. noch nicht möglich, ein Geselligiceitsprofiramm festzulegen. Doch sind entsprechende Vorbereitungen ge-troffen. Auch dieZeit derDamenderTagungsteilnehmer wird ausgefüllt sein.

Die Mitnahme eines Passes mit Lichtbild ist für alle Fälle ratsam.

Die Mitglieder des Vorstandes werden hierdurch zu einer am Dienstag, den 22. Mai 1923, vormittags $10 \mathrm{Uhr}$, in der Wohnung des 1 . Vorsitzenden, Zeppelinstraße 33, stattfindenden Vorstandssitzung eingeladen.

Die Vereinigung zur Förderung des Hebammenwesens wird ihre Sitzung am Dienstag, den 22. Mai 1923, um 4 Uhr nachmittags, im Hörsaal der Universitäts-Frauenklinik abhalten. Das Sitzungsprogramm wird in der endgültigen Kongreßtagesordnung bekanntgegeben.

Merge, 1. Vorsitzender. 